\title{
AARP
}

\section{AARP ElderWatch}

a partnership with the Colorado Attorney General

\section{Finances and Coloradans}

Annotated Questionnaire

Screening Criteria: Age 50+, resident of Colorado, involved in household financial decisions 17.5 minutes

Total sample $n=817$

Fielded March 26 - April 6, 2021.

The sample has been weighted by education based on 2019 4-yr CPS statistics.

For each survey question, this annotated questionnaire displays the distribution of responses among all respondents who were asked the question. The detailed report about these findings may be found at www.aarp.org/COfinances50. For certain questions, the results displayed in the detailed report may look different from the results presented in this annotated questionnaire. Differences are due to the fact that the report at times presents the results for only certain subsets of respondents (such as after excluding respondents who indicated that a question did not apply to them). Questions for which the detailed report displays results among a subset of respondents are marked with two asterisks in this annotated questionnaire.

\section{Screener}

S1. Our study is interested in the opinions of certain age groups. Could you please tell me your age as of your last birthday? [IN YEARS] (TERMINATE IF NOT AGE 50 OR OLDER, OR IF REFUSED)

\begin{tabular}{|l|l|}
\hline$\%$ & $\mathrm{n}=817$ \\
\hline 59 & $50-64$ \\
\hline 25 & $65-74$ \\
\hline 17 & $75+$ \\
\hline
\end{tabular}

S2. And just to confirm, are you a resident of Colorado?

\begin{tabular}{|c|l|}
\hline$\%$ & $\mathrm{n}=817$ \\
\hline 100 & Yes \\
\hline 0 & No (TERMINATE) \\
\hline 0 & Don't know/refused (TERMINATE) \\
\hline
\end{tabular}

S3. What is your 5-digit ZIP code? (TERMINATE IF NO ONE IN HH IS AGE 50+ RESIDENT OF COLORADO) 
S4. As I read each of the following financial tasks for your household, please tell me if you are the primary decision maker for your household, or is another household member the primary decision maker, or do you and another household member share in the decisions? The first is [XXX].

\begin{tabular}{|l|c|c|c|c|}
\hline \multicolumn{1}{|c|}{$\mathrm{n}=817$} & $\begin{array}{c}\text { Another } \\
\text { I am the } \\
\text { primary } \\
\text { decision maker } \\
\%\end{array}$ & $\begin{array}{c}\text { memsehold } \\
\text { share in the } \\
\text { decisions } \\
\%\end{array}$ & $\begin{array}{c}\text { Another } \\
\text { household } \\
\text { member is the } \\
\text { primary decision } \\
\text { maker } \\
\%\end{array}$ & $\begin{array}{c}\text { Don't know/ } \\
\text { refused } \\
\%\end{array}$ \\
\hline Pay monthly bills & 63 & 33 & 4 & $<1$ \\
\hline $\begin{array}{l}\text { Shop/Make purchases } \\
\text { for the household }\end{array}$ & 55 & 41 & 5 & $<1$ \\
\hline $\begin{array}{l}\text { Make decisions about } \\
\text { savings and investing }\end{array}$ & 57 & 44 & 0 & 0 \\
\hline $\begin{array}{l}\text { Make decisions about } \\
\text { other financial issues }\end{array}$ & 52 & 47 & 1 & 0 \\
\hline
\end{tabular}

[Terminate if not "primary" or "share" for bills or shopping, and saving/investing] Just to clarify - they MUST be primary or share for saving/investing (item 3) and they must also be primary or share for either pay monthly bills (item 1) OR shop (item 2)]

SD5. To ensure it is recorded accurately, could you please state your gender?

\begin{tabular}{|c|l|}
\hline$\%$ & $\mathrm{n}=817$ \\
\hline 46 & Male \\
\hline 53 & Female \\
\hline 0 & Other \\
\hline$<1$ & Refused \\
\hline
\end{tabular}

$\underline{\underline{\text { SD6 }}}$. Which statement best describes your current employment status? [Read list]

\begin{tabular}{|c|l|}
\hline$\%$ & $\mathrm{n}=817$ \\
\hline 33 & Working - as a paid employee \\
\hline 14 & Self-employed \\
\hline 1 & Not working, on temporary layoff from a job \\
\hline 3 & Not working, but looking for work \\
\hline 39 & Not working, and completely retired \\
\hline 6 & Not working and disabled \\
\hline 5 & $\begin{array}{l}\text { Not working for another reason (if needed: homemaker, student, or } \\
\text { another reason) }\end{array}$ \\
\hline 0 & Refused [Do not read] \\
\hline
\end{tabular}


If SD6 = not working and disabled or not working for another reason:

SD8. Do you consider yourself to be retired?

\begin{tabular}{|c|l|}
\hline$\%$ & $\mathrm{n}=76$ \\
\hline 51 & Yes \\
\hline 47 & No \\
\hline 2 & Refused \\
\hline
\end{tabular}

SD9. What is your current marital status? [Read list]

\begin{tabular}{|c|l|}
\hline$\%$ & $\mathrm{n}=817$ \\
\hline 54 & Married \\
\hline 4 & Not married, living with your partner or significant other \\
\hline 2 & Separated \\
\hline 14 & Divorced \\
\hline 14 & Widowed \\
\hline 12 & Currently single and never married \\
\hline 1 & Refused [Do not read] \\
\hline
\end{tabular}

\section{PART A: FINANCIAL EDUCATION AND REGRETS}

A1. As I read each of the following, please tell me if it has had a major influence, a minor influence, or no influence at all on your approach to your money and personal finances over the course of your life?

[Randomize]

\begin{tabular}{|l|c|c|c|c|}
\hline \multicolumn{1}{|c|}{$\mathrm{n}=817$} & $\begin{array}{c}\text { Major } \\
\text { influence } \\
\%\end{array}$ & $\begin{array}{c}\text { Minor } \\
\text { influence } \\
\%\end{array}$ & $\begin{array}{c}\text { No influence } \\
\text { at all } \\
\%\end{array}$ & $\begin{array}{c}\text { Refused } \\
\%\end{array}$ \\
\hline Parents & 38 & 28 & 33 & 1 \\
\hline Spouse/Partner & 45 & 23 & 31 & 2 \\
\hline Other family members & 12 & 39 & 48 & 1 \\
\hline Friends & 11 & 42 & 47 & $<1$ \\
\hline $\begin{array}{l}\text { School (including teachers, other students, } \\
\text { and information provided at school) }\end{array}$ & 18 & 33 & 48 & 1 \\
\hline $\begin{array}{l}\text { Workplace (including your employer, } \\
\text { coworkers, and information provided at } \\
\text { work) }\end{array}$ & 22 & 34 & 42 & 1 \\
\hline $\begin{array}{l}\text { Internet (such financial web sites, social } \\
\text { media, internet searches) }\end{array}$ & 12 & 35 & 52 & 1 \\
\hline Books, magazines, or newspapers & 14 & 41 & 44 & 1 \\
\hline TV or radio & 9 & 39 & 52 & $<1$ \\
\hline Podcasts & 2 & 11 & 84 & 3 \\
\hline $\begin{array}{l}\text { Financial advisors or other financial } \\
\text { professionals }\end{array}$ & 26 & 35 & 39 & 1 \\
\hline Your bank or investment firm & 22 & 39 & 39 & 1 \\
\hline Other (specify) & $<1$ & $<1$ & 96 & 3 \\
\hline $\begin{array}{l}\text { Prayer, church, or faith (unaided from other- } \\
\text { specify comments) }\end{array}$ & 2 & $<1$ & 98 & \\
\hline
\end{tabular}


A2. Who or what has had the most influence on your approach to your money and personal finances? (Select one.) (Read list; include “Don't know” and “Refused” options)

\begin{tabular}{|c|l|}
\hline$\%$ & n=817 \\
\hline 19 & Parents \\
\hline 29 & Spouse/Partner \\
\hline 3 & Other family members \\
\hline 2 & Friends \\
\hline 2 & $\begin{array}{l}\text { School (including teachers, other students, and information provided at } \\
\text { school) }\end{array}$ \\
\hline 6 & $\begin{array}{l}\text { Workplace (including your employer, coworkers, and information provided } \\
\text { at work) }\end{array}$ \\
\hline 4 & Internet (such financial web sites, social media, internet searches) \\
\hline 5 & Books, magazines, or newspapers \\
\hline 2 & TV or radio \\
\hline$<1$ & Podcasts \\
\hline 14 & Financial advisors or other financial professionals \\
\hline 4 & Your bank or investment firm \\
\hline 1 & Prayer, church, or faith (unaided from other-specify comments) \\
\hline$<1$ & Other (previously specified in A1) \\
\hline 3 & Don't know \\
\hline 1 & Refused \\
\hline 4 & Not asked - all A1 series items "not a reason" or "refused" \\
\hline
\end{tabular}

A3. At roughly what age did you first begin learning important lessons about money and personal finances? [Only read response options if necessary]

\begin{tabular}{|c|l|}
\hline$\%$ & n=817 \\
\hline 27 & As a young child (12 or younger) \\
\hline 39 & As a teenager (13-19) \\
\hline 18 & In your 20s \\
\hline 9 & 30 s \\
\hline 4 & 40 s \\
\hline 2 & 50 s \\
\hline$<1$ & 60 s \\
\hline 0 & 70 s \\
\hline 0 & 80 s or older \\
\hline$<1$ & Never - I do not know anything about personal finances \\
\hline 1 & Don't know [Do not read] \\
\hline 0 & Refused [Do not read] \\
\hline
\end{tabular}


$* *$ A4. People sometimes think back on the course of their life and wish they had made different choices about their money and personal finances. For each area of your finances that I'm about to mention, please tell me if you think you (if married: and your spouse) have made...? [Reverse order for random 50\%] [Read list]

\begin{tabular}{|l|c|c|c|c|c|c|c|c|}
\hline \multicolumn{1}{|c|}{$\mathrm{n}=817^{* *}$} & $\begin{array}{c}\text { All } \\
\text { good } \\
\%\end{array}$ & $\begin{array}{c}\text { Mostly } \\
\text { good } \\
\%\end{array}$ & $\begin{array}{c}\text { Mix of } \\
\text { good } \\
\text { and } \\
\text { poor } \\
\%\end{array}$ & $\begin{array}{c}\text { Mostly } \\
\text { poor } \\
\%\end{array}$ & $\begin{array}{c}\text { All poor } \\
\%\end{array}$ & $\begin{array}{c}\text { N/A } \\
\text { [Do not } \\
\text { read] } \\
\%\end{array}$ & $\begin{array}{c}\text { Don't } \\
\text { know } \\
\text { [Do not } \\
\text { read] } \\
\%\end{array}$ & $\begin{array}{c}\text { Ref. } \\
\text { [Do not } \\
\text { read] } \\
\%\end{array}$ \\
\hline Saving for retirement & 24 & 42 & 17 & 10 & 5 & 2 & $<1$ & 0 \\
\hline $\begin{array}{l}\text { Buying a house and } \\
\text { home ownership }\end{array}$ & 50 & 35 & 7 & 2 & 1 & 4 & $<1$ & $<1$ \\
\hline $\begin{array}{l}\text { Buying a car and car } \\
\text { ownership }\end{array}$ & 35 & 47 & 12 & 3 & $<1$ & 3 & $<1$ & 0 \\
\hline $\begin{array}{l}\text { Major purchases other } \\
\text { than a house or car }\end{array}$ & 26 & 49 & 16 & 3 & 1 & 5 & 1 & $<1$ \\
\hline Managing your debt & 34 & 36 & 19 & 5 & 2 & 3 & $<1$ & $<1$ \\
\hline $\begin{array}{l}\text { Spending within your } \\
\text { means }\end{array}$ & 32 & 42 & 20 & 4 & 2 & $<1$ & $<1$ & $<1$ \\
\hline $\begin{array}{l}\text { Saving for future } \\
\text { emergencies or } \\
\text { unexpected expenses }\end{array}$ & 25 & 42 & 21 & 8 & 3 & 2 & 0 & 0 \\
\hline $\begin{array}{l}\text { Loaning money to } \\
\text { others }\end{array}$ & 25 & 29 & 14 & 9 & 6 & 17 & $<1$ & $<1$ \\
\hline $\begin{array}{l}\text { Saving for children's } \\
\text { education }\end{array}$ & 18 & 26 & 15 & 9 & 4 & 28 & 1 & $<1$ \\
\hline $\begin{array}{l}\text { Investment choices } \\
\text { like what you invested } \\
\text { your money in) }\end{array}$ & 17 & 43 & 23 & 6 & 2 & 8 & 1 & $<1$ \\
\hline
\end{tabular}

** The results displayed in this table are among all 817 respondents, including those who indicated that an item was not applicable to them. In contrast, the results displayed in the report have been rebased to exclude respondents who indicated that an item was not applicable.

\section{PART B: RETIREMENT}

B1. Have you (if married: or your spouse) ever saved for retirement?

\begin{tabular}{|l|l|}
\hline$\%$ & $\mathrm{n}=817$ \\
\hline 86 & Yes \\
\hline 14 & No \\
\hline$<1$ & Don't know \\
\hline$<1$ & Refused \\
\hline
\end{tabular}




\section{If not retired:}

**B2. Are you (if married: or your spouse) currently saving for retirement?

\begin{tabular}{|c|l|}
\hline$\%$ & $\mathrm{n}=460^{* *}$ \\
\hline 82 & Yes \\
\hline 18 & No \\
\hline 0 & Don't know \\
\hline$<1$ & Refused \\
\hline
\end{tabular}

**The above table displays results among all non-retired respondents, including those who are currently working and those who are not working. In contrast, the report displays results only among those who are working.

\section{If B1=yes:}

B3. At what age did you first start saving for retirement? (Your best guess is fine.) [Record answer; ensure that answer is not older than respondent's current age]

\begin{tabular}{|c|l|}
\hline$\%$ & $\mathrm{n}=715$ \\
\hline 24 & $<25$ \\
\hline 37 & $25-34$ \\
\hline 23 & $35-44$ \\
\hline 15 & $45+$ \\
\hline 2 & Don't know/refused \\
\hline 32.1 & Mean \\
\hline 30.1 & Median \\
\hline
\end{tabular}

All:

**B4. At what age do you wish you had started saving for retirement? [Record answer]

\begin{tabular}{|c|l|}
\hline$\%$ & $\mathrm{n}=817^{* *}$ \\
\hline 54 & $<25$ \\
\hline 27 & $25-34$ \\
\hline 8 & $35-44$ \\
\hline 4 & $45+$ \\
\hline 8 & Don't know/refused \\
\hline 23.7 & Mean \\
\hline 21.3 & Median \\
\hline
\end{tabular}

** The above table displays results among all respondents. The report displays results two ways among all respondents as well as only among respondents who stated that they had ever saved for retirement (B1=yes). 
B6. Have you (if married: or your spouse) ever made regular contributions to any of the following retirement savings tools...? (READ LIST) [ROTATE A-B/B-A, READ C LAST]

\begin{tabular}{|l|c|c|c|c|}
\hline \multicolumn{1}{|c|}{$\mathrm{n}=817$} & $\begin{array}{c}\text { Yes } \\
\%\end{array}$ & $\begin{array}{c}\text { No } \\
\%\end{array}$ & $\begin{array}{c}\text { Not sure } \\
\%\end{array}$ & $\begin{array}{c}\text { Refused } \\
\%\end{array}$ \\
\hline $\begin{array}{l}\text { A workplace retirement saving plan, such as a } \\
\text { 401(k) or 403(b) or IRA, offered by your employer }\end{array}$ & 74 & 26 & 1 & $<1$ \\
\hline $\begin{array}{l}\text { A personal retirement savings account, such as an } \\
\text { IRA, that you have outside of work }\end{array}$ & 55 & 44 & 1 & $<1$ \\
\hline Something else to help you save for retirement & 50 & 49 & 1 & $<1$ \\
\hline
\end{tabular}

If employed as a paid employee (SD6=1):

B7. Which of the following types of retirement plans or ways to save for retirement does your current employer provide...do they provide? [MULTIPLE RESPONSES ALLOWED - ROTATE A AND B] [READ EACH ANSWER CATEGORY]

\begin{tabular}{|c|l|}
\hline$\%$ & $\mathrm{n}=279$ \\
\hline 30 & $\begin{array}{l}\text { A traditional pension plan- a defined benefit plan? [lf asked, a defined } \\
\text { benefit plan supplies retirees with a monthly income, typically d on a } \\
\text { formula of salary and years of service. It is not a 401( } \mathrm{k}) \text { plan] }\end{array}$ \\
\hline 73 & $\begin{array}{l}\text { An IRA, 401k or 403b retirement savings plan? [If asked, these plans } \\
\text { allow you to make contributions from your salary to an individual account } \\
\text { set up in your name] }\end{array}$ \\
\hline 17 & Or employer does NOT offer a way to save for retirement \\
\hline 1 & Don't know [Do not read] \\
\hline 1 & Refused [Do not read] \\
\hline
\end{tabular}

If not retired (including those who are employed as well as those who are not employed but not retired): **B8. Are you currently making regular contributions to any of the following savings tools...? (READ LIST) [ROTATE A-B/B-A, READ C LAST]

\begin{tabular}{|l|c|c|c|c|}
\hline \multicolumn{1}{|c|}{$\mathrm{n}=460 * *$} & $\begin{array}{c}\text { Yes } \\
\%\end{array}$ & $\begin{array}{c}\text { No } \\
\%\end{array}$ & $\begin{array}{c}\text { Not sure } \\
\%\end{array}$ & $\begin{array}{c}\text { Refused } \\
\%\end{array}$ \\
\hline $\begin{array}{l}\text { [If existing employer offers an IRA, 401(k) or } \\
\text { 403(b); } \mathrm{n=204]} \mathrm{A} \mathrm{workplace} \mathrm{retirement} \mathrm{saving} \\
\text { plan such as a 401(k), 403(b), or IRA offered by } \\
\text { your employer }\end{array}$ & 84 & 16 & 0 & 0 \\
\hline $\begin{array}{l}\text { [All] A personal retirement savings account such } \\
\text { as an IRA that you have outside of work }\end{array}$ & 39 & 58 & 1 & 3 \\
\hline $\begin{array}{l}\text { [All] Something else to help you save for } \\
\text { retirement }\end{array}$ & 42 & 54 & 1 & 3 \\
\hline
\end{tabular}

**The above table displays results among all non-retired respondents, including those who are not currently working as well as those who are self-employed. In contrast, the report displays results for this question calculated only among non-retired respondents who are currently working as paid employees. 


\section{If not retired:}

B10. Realistically, at what age do you expect to retire? [If needed: If you expect to retire gradually, at what age do you expect you will begin to retire?]

\begin{tabular}{|c|l|}
\hline$\%$ & $\mathrm{n}=460$ \\
\hline 7 & $<60$ \\
\hline 35 & $60-65$ \\
\hline 27 & $66-70$ \\
\hline 21 & $71+/$ will never retire \\
\hline 10 & Don't know/refused \\
\hline 66.3 & Mean \\
\hline 65.4 & Median \\
\hline
\end{tabular}

\section{If retired:}

B11. How old were you when you retired? [If needed: If you retired gradually, how old were you when you began to retire?]

\begin{tabular}{|c|l|}
\hline$\%$ & $\mathrm{n}=357$ \\
\hline 36 & $<60$ \\
\hline 42 & $60-65$ \\
\hline 10 & $66-70$ \\
\hline 6 & $71+$ \\
\hline 7 & Never worked/don't know/refused \\
\hline 60.0 & Mean \\
\hline 61.4 & Median \\
\hline
\end{tabular}

\section{If retired:}

B12. Did you retire...? [RANDOMLY REVERSE LIST; read list]

\begin{tabular}{|c|l|}
\hline$\%$ & $\mathrm{n}=357$ \\
\hline 43 & Earlier than planned \\
\hline 44 & About when planned \\
\hline 8 & Later than planned \\
\hline 4 & Don't know [Do not read] \\
\hline 1 & Refused [Do not read] \\
\hline
\end{tabular}


If retired, and retired earlier than planned:

B13. Did you retire earlier than you planned because...? [RANDOMIZE. KEEP E LAST]

\begin{tabular}{|l|c|c|c|c|}
\hline \multicolumn{1}{|c|}{$\mathrm{n}=149$} & Yes & $\begin{array}{c}\text { No } \\
\%\end{array}$ & $\begin{array}{c}\text { Don't } \\
\text { know [Do } \\
\text { not read] } \\
\%\end{array}$ & $\begin{array}{c}\text { Refused } \\
\text { [Do not } \\
\text { read] } \\
\%\end{array}$ \\
\hline You could afford to retire earlier than you planned & 47 & 52 & 1 & 1 \\
\hline You had a health problem or a disability & 49 & 50 & 1 & 0 \\
\hline $\begin{array}{l}\text { There were changes at your company, such as } \\
\text { downsizing, closure or reorganization }\end{array}$ & 30 & 68 & 0 & 2 \\
\hline $\begin{array}{l}\text { Because of changes in the skills required for your job } \\
\text { or your skills no longer matching job requirements }\end{array}$ & 18 & 80 & 2 & 0 \\
\hline You wanted to do something else & 26 & 73 & 0 & 1 \\
\hline You had to care for a spouse or another family member & 22 & 76 & 1 & 1 \\
\hline You had another work-related reason (specify) & 6 & 93 & 0 & 1 \\
\hline
\end{tabular}

Ask all:

B14. [If not retired, add: "Let's assume that at some point you (if married: "and your spouse") will have to fully retire and stop working completely."]

How likely are you to have enough money to cover your needs throughout your retirement if you (if married: "and your spouse") had to rely only on your savings, investments, and Social Security and were not able to work? [READ LIST]

\begin{tabular}{|c|l|}
\hline$\%$ & $\mathrm{n}=817$ \\
\hline 28 & Extremely likely \\
\hline 30 & Very likely \\
\hline 25 & Somewhat likely \\
\hline 8 & Not too likely \\
\hline 7 & Not at all likely \\
\hline 2 & Don't know [Do not read] \\
\hline$<1$ & Refused [Do not read] \\
\hline
\end{tabular}

\section{PART C: PAST 12 MONTHS AND EVERYDAY FINANCIAL BEHAVIORS}

C1A. Overall, how satisfied are you with your overall financial situation? Are you: [Read list]

\begin{tabular}{|c|l|}
\hline$\%$ & n=817 \\
\hline 19 & Extremely satisfied \\
\hline 29 & Very satisfied \\
\hline 37 & Somewhat satisfied \\
\hline 11 & Not too satisfied \\
\hline 5 & Not at all satisfied \\
\hline$<1$ & Don't know [Do not read] \\
\hline$<1$ & Refused [Do not read] \\
\hline
\end{tabular}


C1. Compared to this time last year, (that is, March 2020), would you say that each of the following is now better, worse, or about the same. The first is [XXX] [Randomize first three]

\begin{tabular}{|l|c|c|c|c|c|}
\hline \multicolumn{1}{|c|}{$\mathrm{n}=817$} & $\begin{array}{c}\text { Better } \\
\text { now } \\
\%\end{array}$ & $\begin{array}{c}\text { Worse } \\
\text { now } \\
\%\end{array}$ & $\begin{array}{c}\text { About } \\
\text { the } \\
\text { same } \\
\%\end{array}$ & $\begin{array}{c}\text { Don't } \\
\text { know [Do } \\
\text { not read] } \\
\%\end{array}$ & $\begin{array}{c}\text { Refused } \\
\text { [Do not } \\
\text { read] } \\
\%\end{array}$ \\
\hline $\begin{array}{l}\text { The amount of your total debt (f needed: } \\
\text { including credit cards, mortgage, student } \\
\text { loans, and other debt) }\end{array}$ & 37 & 8 & 53 & 2 & 1 \\
\hline $\begin{array}{l}\text { The amount of savings that you have for } \\
\text { retirement }\end{array}$ & 37 & 12 & 50 & $<1$ & 1 \\
\hline $\begin{array}{l}\text { The amount of savings that you have for } \\
\text { emergencies or unexpected expenses }\end{array}$ & 32 & 12 & 56 & $<1$ & $<1$ \\
\hline Your overall financial situation & 35 & 12 & 53 & $<1$ & 0 \\
\hline
\end{tabular}

C5. In the past 12 months, have you used any peer-to-peer payment platforms to pay another person or make a purchase? (These include PayPal, Venmo, Zelle, Square, Apple Pay, Google Pay, Cash App, and sending payments through Facebook Messenger.)

\begin{tabular}{|c|l|}
\hline$\%$ & $\mathrm{n}=817$ \\
\hline 38 & Yes \\
\hline 61 & No \\
\hline 1 & Not sure \\
\hline 0 & Refused \\
\hline
\end{tabular}

If C5=yes:

C6. Compared to this time last year (that is, March 2020), are you now using peer-to-peer payment platforms more, less, or about the same?

\begin{tabular}{|c|l|}
\hline$\%$ & $\mathrm{n}=330$ \\
\hline 27 & More \\
\hline 8 & Less \\
\hline 65 & About the same \\
\hline$<1$ & Don't know \\
\hline 0 & Refused \\
\hline
\end{tabular}

C7. In the past 12 months, have you purchased merchandise online through a web site or app not affiliated with your bank? (This includes online purchases through Amazon or other retail web sites.)

\begin{tabular}{|c|l|}
\hline$\%$ & $\mathrm{n}=817$ \\
\hline 72 & Yes \\
\hline 28 & No \\
\hline$<1$ & Not sure \\
\hline 0 & Refused \\
\hline
\end{tabular}


If C7=yes:

C8. Compared to this time last year (that is, March 2020), are you now purchasing merchandise online more, less, or about the same?

\begin{tabular}{|c|l|}
\hline$\%$ & $\mathrm{n}=611$ \\
\hline 36 & More \\
\hline 9 & Less \\
\hline 54 & About the same \\
\hline$<1$ & Don't know \\
\hline 0 & Refused \\
\hline
\end{tabular}

C13. In the past 12 months, have you checked your free annual credit report from www.annualcreditreport.com or Credit Karma or another way? [If needed: A credit report shows all your loans and debts, including credit cards, and your payments on those debts. It is not just your credit score.]

\begin{tabular}{|c|l|}
\hline$\%$ & $\mathrm{n}=817$ \\
\hline 48 & Yes \\
\hline 52 & No \\
\hline$<1$ & You checked your credit report in the past year, but you had to pay for it \\
\hline$<1$ & Don't know \\
\hline 0 & Refused \\
\hline
\end{tabular}

C15. Have you ever lost money as part of what you consider to be a scam or fraud?

\begin{tabular}{|c|l|}
\hline$\%$ & $\mathrm{n}=817$ \\
\hline 26 & Yes \\
\hline 71 & No \\
\hline 1 & I think so \\
\hline 1 & Don't know \\
\hline 0 & Refused \\
\hline
\end{tabular}

\section{PART D: PREPARING FOR THE FUTURE}

D1. In the past year, have you discussed your finances with [if married/partnered: "your spouse or partner or any other loved ones" / if not married/not partnered: "any of your loved ones"]? This could include emails, texts, phone calls, or in-person conversations about financial matters such as spending, savings, and/or your financial preparations for the future.

\begin{tabular}{|l|l|}
\hline$\%$ & $\mathrm{n}=817$ \\
\hline 74 & Yes \\
\hline 25 & No \\
\hline$<1$ & Don't know \\
\hline$<1$ & Refused \\
\hline
\end{tabular}




\section{If D1=no:}

D2. Which of the following best describes why you have not discussed your finances with any of your loved ones in the past year? [Randomize; read list; select one]

\begin{tabular}{|c|l|}
\hline$\%$ & n=193 \\
\hline 35 & Your finances are none of their business \\
\hline 1 & You would be embarrassed to discuss your finances with them \\
\hline 8 & They would not be interested \\
\hline 44 & You don't see a reason to discuss your finances with them \\
\hline 4 & You don't know if you can trust them \\
\hline 3 & You don't have any loved ones that you feel close to \\
\hline 3 & Another reason (specify) \\
\hline 2 & Don't know [Do not read] \\
\hline 1 & Refused [Do not read] \\
\hline
\end{tabular}

D3. Do you have any money set aside that you consider savings for emergencies?

\begin{tabular}{|c|l|}
\hline$\%$ & $\mathrm{n}=817$ \\
\hline 85 & Yes \\
\hline 14 & No \\
\hline$<1$ & Don't know \\
\hline 0 & Refused \\
\hline
\end{tabular}

D3A. How confident are you that you would be able to pay for an emergency or unexpected expense in the amount of $\$ 2,000$ without [if not retired: "taking money from your retirement accounts"] If retired: "risking your ability to cover your other living expenses"]? [Read list]

\begin{tabular}{|c|l|}
\hline$\%$ & $\mathrm{n}=817$ \\
\hline 54 & Extremely confident \\
\hline 20 & Very confident \\
\hline 13 & Somewhat confident \\
\hline 4 & Not too confident \\
\hline 8 & Not at all confident \\
\hline$<1$ & Don't know [Do not read] \\
\hline$<1$ & Refused [Do not read] \\
\hline
\end{tabular}

D4. Do you have your important financial and health-related information stored in a safe location that a loved one can access in case of an emergency? (Such information may include bank account information, passwords, tax information, names of your doctors, any other important legal documents, etc.)

\begin{tabular}{|c|l|}
\hline$\%$ & $\mathrm{n}=817$ \\
\hline 49 & Yes, all of this information \\
\hline 23 & Yes, most of this information \\
\hline 12 & Yes, some of this information \\
\hline 14 & No, none \\
\hline 1 & Don't know [Do not read] \\
\hline 1 & Refused [Do not read] \\
\hline
\end{tabular}


D5. Have you done any of the following?

\begin{tabular}{|l|c|c|c|c|}
\hline \multicolumn{1}{|c|}{$\mathrm{n}=817$} & $\begin{array}{c}\text { Yes } \\
\%\end{array}$ & $\begin{array}{c}\text { No } \\
\%\end{array}$ & $\begin{array}{c}\text { Don't } \\
\text { know } \\
\%\end{array}$ & $\begin{array}{c}\text { Refused } \\
\%\end{array}$ \\
\hline $\begin{array}{l}\text { Prepared a last will and testament that controls } \\
\text { how your money and other property are to be } \\
\text { distributed }\end{array}$ & 62 & 38 & $<1$ & $<1$ \\
\hline $\begin{array}{l}\text { Prepared a living will or other written instructions } \\
\text { in which you state the kind of healthcare you } \\
\text { want or don't want under certain conditions }\end{array}$ & 61 & 38 & 1 & $<1$ \\
\hline $\begin{array}{l}\text { Appointed someone as a financial power of } \\
\text { attorney to make financial decisions on your } \\
\text { behalf if you become unable to }\end{array}$ & 59 & 41 & 1 & $<1$ \\
\hline
\end{tabular}

\section{If no to all parts of D5:}

D6. Which of the following best describes why you don't have a will, a living will, or a healthcare proxy? Is it because you...? [Randomize; read list] (Single Select)

\begin{tabular}{|c|l|}
\hline$\%$ & n=195 \\
\hline 9 & Don't think you need any of them \\
\hline 4 & Don't know how to go about preparing them \\
\hline 8 & Never thought about it \\
\hline 70 & Thought about it but haven't gotten around to it \\
\hline 7 & Think it might cost too much \\
\hline 0 & Other (specify) [Do not read] \\
\hline 1 & Don't know [Do not read] \\
\hline 1 & Refused [Do not read] \\
\hline
\end{tabular}

\section{Part E: Additional Profile Questions/Demographics}

E2. What is the highest level of education that you have completed? [READ LIST IF NECESSARY]

\begin{tabular}{|c|l|}
\hline$\%$ & $\mathrm{n}=817$ \\
\hline 5 & Less than a high school degree \\
\hline 24 & High school graduate \\
\hline 14 & Some college but less than two years \\
\hline 16 & Some college two years or more/AA degree \\
\hline 21 & College graduate/bachelor's degree/BA/BS \\
\hline 4 & Postgraduate courses \\
\hline 17 & Graduate or professional degree \\
\hline$<1$ & Don't know/none specified [Do not read] \\
\hline
\end{tabular}


E5. For statistical purposes only, in which of the following categories was your total household income for 2020? [READ CHOICES]

\begin{tabular}{|c|l|}
\hline$\%$ & $\mathrm{n}=817$ \\
\hline 16 & Less than $\$ 30,000$ \\
\hline 10 & $\$ 30,000$ to less than $\$ 40,000$ \\
\hline 10 & $\$ 40,000$ to less than $\$ 50,000$ \\
\hline 16 & $\$ 50,000$ to less than $\$ 75,000$ \\
\hline 13 & $\$ 75,000$ to less than $\$ 100,000$ \\
\hline 12 & $\$ 100,000$ to less than $\$ 150,000$ \\
\hline 12 & $\$ 150,000$ or more \\
\hline 11 & Don't know/refused [Do not read] \\
\hline
\end{tabular}

E6. Are you [if married: "or your spouse"/if partnered: "or partner"] a member of A-A-R-P?

\begin{tabular}{|c|l|}
\hline$\%$ & n=817 \\
\hline 39 & Yes \\
\hline 59 & No \\
\hline 2 & Don't know \\
\hline$<1$ & Refused \\
\hline
\end{tabular}

E7. The next two questions are for statistical purposes only. Are you of Hispanic, Spanish, or Latino origin or descent?

\begin{tabular}{|c|l|}
\hline$\%$ & $\mathrm{n}=817$ \\
\hline 13 & Yes \\
\hline 85 & No \\
\hline$<1$ & Don't know \\
\hline 2 & Refused \\
\hline
\end{tabular}

E8. What is your race? Are you... [READ LIST]? [MULTIPLE RESPONSES ALLOWED]

\begin{tabular}{|c|l|}
\hline$\%$ & $\mathrm{n}=817$ \\
\hline 86 & White or Caucasian \\
\hline 4 & Black or African American \\
\hline 1 & Asian \\
\hline 1 & Native Hawaiian or Other Pacific Islander \\
\hline 3 & American Indian or Alaska Native \\
\hline 4 & Other (specify) \\
\hline 3 & Don't know/ prefer not to answer [Do not read] \\
\hline
\end{tabular}

